\title{
Transverse Myelitis Associated to HCV Infection
}

\begin{abstract}
Diego Michelon De Carli ${ }^{1}$, Jeferson Pannebeker ${ }^{1}$, Fábio Lopes Pedro ${ }^{2}$, Carlos Jesus Pereira Haygert ${ }^{3}$, Everaldo Hertz ${ }^{4}$ and $^{\circ}$ Maristela de Oliveira Beck ${ }^{5}$

${ }^{1}$ Médicos Residentes de Clinica Médica Hospital Universitário de Santa Maria; ${ }^{2}$ Médico Residente de Infectologia Hospital Universitário de Santa Maria; ${ }^{3}$ Médico Radiologista do Hospital Universitário de Santa Maria; ${ }^{4}$ Médico Oncologista, Preceptor da Residência de Clinica Médica do Hospital Universitário de Santa Maria; ${ }^{5}$ Médica Endocrinologista, Chefe do Serviço de Clinica Médica Hospital Universitário de Santa Maria; Santa Maria, RS, Brazil
\end{abstract}

\begin{abstract}
Complications involving the central nervous system in patients suffering from hepatitis C virus (HCV) infection have been rare. Among them, it appears the transverse myelitis, which has already been reported in likely association with HCV. This paper presents the case study of a 65-year-old woman who developed cervical transverse myelitis linked to chronic HCV infection and anti-HCV antibodies in the spinal fluid, being excluded other etiologies for transverse myelitis. Current evidence has reinforced the likely association between chronic HCV infection and transverse myelitis, especially as recurrent manifestations of the disease. These findings reveal the need for more searching to establish the causal relationship between transverse myelitis and hepatitis $\mathrm{C}$.
\end{abstract}

Key-Words: Central Nervous System, HCV infection, transverse myelitis.

The World Health Organization estimates that infection by the hepatitis $\mathrm{C}$ virus (HCV) affects around $3 \%$ of the world population [1], being one of the main causes of the chronic hepatic disease. In HCV-positive patients, extra-hepatic symptoms are reported in up to $74 \%$ of the cases, $10 \%$ to $15 \%$ of which regarding the neurological changes, mainly in the peripheral nervous system, and in this context associated to the cryoglobulinaemia. Manifestations observed in the central nervous system (CNS) include chronic forms of encephalopathy, epilepsy, normal pressure hydrocephaly, ischemic encephalovascular syndrome, Ekbom syndrome, and encephalomyelitis [2-5].

Transverse myelitis (TM) is a demyelinating disease of the spinal cord. The disease is characterized by the emergence of acute or sub acute signs and symptoms of motor, neurological, and autonomic dysfunction related to the nervous tracts of the spinal cord [6]. TM often occurs due to inflammatory and infectious processes, rheumatologic, neoplastic, iatrogenic, demyelinating, compressive, actinic as well as idioapthic diseases [7,8]. TM associated to HCV infection has recently been reported, and although the causal mechanism has not been fully established yet, it is hypothesized that the virus may act directly in the development of TM, or lead to immune-mediated injury.

This paper is a case study of a patient who suffered from cervico-dorsal spinal cord myelitis, In association with updated literature, this study case adds evidence about the association between HCV and TM.

Received on 6 October July; revised 25 February 2009.

Address for correspondence: Dr. Diego De Carli. Universidade Federal de Santa Maria (UFSM), Hospital Universitário de Santa Maria (HUSM), Serviço de Clinica Médica do HUSM. Rua dos Andradas nº1125/301, Bairro Centro. Zip code: 97010-031. Santa Maria-RS, Brazil. E-mail: de.carli@terra.com.br. Phone: (55) 99443827.

The Brazilian Journal of Infectious Diseases

2009;13(2):147-152. (C) 2009 by The Brazilian Journal of Infectious Diseases and Contexto Publishing. All rights reserved.

\section{Case Report}

The patient was a nulliparous, non-smoker 65-year-old caucasian woman previously alcoholic but abstemious for eight years, with obesity degree 3, hypertension, diabetes mellitus type II, and with a metallic mitral valve for nine months. The patient was from the central region of the state of Rio Grande do Sul, Brazil, and had been taking warfarin, metphormin, glibenclamide, hydrochlothiazide, metoprolol, enalipril, and furosemide at the time of hospital admission.

On admission, complaints reported by the patient included strong and progressive thoracolumbar pain for 30 days, which evolved with the loss of strength and sensitivity in lower limbs five days prior to admission. The patient also presented urinary retention, constipation for nearly seven days, and diffuse abdominal pain. On the physical examination, the patient felt reasonably well, lucid and coherent. The examination also revealed that the patient was eupneic, with $\mathrm{HR}=82 \mathrm{bpm}$ and $\mathrm{BP}=110 / 75$ paraplegic with a sensitive level on the navel and coetaneous-plantar extensor reflex on the left side. Other observations included distended abdomen with hydro aerial sounds, painful to decompression. Lung exams were uneventful. Cardiac auscultation revealed unchanged valve click. Laboratory exams results have been showed in Table 1. Urine sediments revealed: leukocyte esterase: positive +++; nitrite: positive; erythrocytes: +50 per field; leukocytes: +50 per field. Urine culture with Escherichia coli was $>100,000 \mathrm{CFU}$ per field. Radiographs revealed acute abdomen with loop distension. Computer tomography of the abdomen revealed distension of the small and large intestines, apart from porcelain gallbladder. Vesical probing, Fleet mineral oil enema, hydrolectrolytic treatment were carried out, followed by a course of ciprofloxacin and metronidazole. Anticoagulation was promoted by non fractionated heparin, and glycemic control was reached with insulin.

Abdominal pain receded, though the neurological status remained unaltered. Ten days after, urine culture was positive for ciprofloxacin-resistant enterococcus, and the initial antibiotic treatment was replaced by piperacillin/tazobactam. Spinal cord MRI was performed. A spontaneous hyper signal 
was observed in T1, while a weak hyper signal was observed in $\mathrm{T} 2$, at the tenth dorsal vertebra, on the left half of the vertebral canal, with strong gradient echo hypo signal. This change measured $1.5 \times 0.8$, and compressed the medulla to the right of the medium line. The radiology results were compatible with the hypothesis of spinal subdural hematoma. On the other zones, the medulla presented a weak hyper signal without significant expansion. The neurosurgical evaluation did not evince medullar decompression. A conservative treatment approach was chosen, in the light of the risks involved in the discontinuation of anticoagulation therapy (thromboembolism), in a context of a neurological lesion already irreversible.

Due to the constantly high transaminase levels, in spite of the improvement of the abdominal picture, an anti-HCV test was requested. The result was positive, and confirmed by HCV PCR. HBsAg was negative.

Constant, high fever (over $38.5^{\circ} \mathrm{C}$ ) was observed from the 13th day on. Hemocultures were conducted, and vancomycin included in the antibiotic treatment. A transthoracic echocardiography was also performed, revealing the normal operation of the metallic mitral valve, a moderate increase in the left atrium, and a left ventricle hypertrophy with preserved systolic function ( $\mathrm{EF}=75 \%)$.

A neurological examination revealed the loss of muscular strength in the upper limbs, which was followed by loss of sensitivity. On the 18th day in hospital, the patient's general status decayed, presenting quadriplegia, with predominantly diaphragmatic breathing, fever, and stiff neck. The patient was lucid though disoriented, with impaired critical judgment and visual hallucinations. A new esophagic echocardiography revealed the mitral valve working normally.

Three lumbar punctures were carried out, all unsuccessful to obtain spinal fluid. On the 30th day in hospital, the patient underwent MRI of the encephalon and cervico-dorsal vertebral column. The exam revealed extensive hyper signal in T2 in the medulla, starting at the bulbomedullar transition and stretching to the visible sections of the dorsal medulla, without significantly increasing medullar thickness, compatible with myelitis (Figures 1 and 2). Next, serology tests were requested to detect the infectious and rheumatologic causes that relate to TM (Table 2). The patient did not present skin changes, ophthalmologic complaints, thromboembolic events, arthlagia or arthritis, history of previous radiotherapeutic treatment. Likewise, the patient had not taken any interstate trip for the previous 15 years.

The general status of the patient remained poor, with mental confusion and delirium, shallow and diaphragmatic breathing, tachypnea, fever and quadriplegia, though with no changes in the pattern of cardiovascular auscultation. The abdomen exam did not reveal any changes. A sub occipital puncture for the cerebrospinal fluid was carried out, being 5 $\mathrm{mL}$ of which sent to immunological, microbiological, cytological and biochemical analyses (Table 2).
On the 56th day in hospital, the patient's sensory status worsened (Glasgow scale 7), with hypotension, and eventually respiratory failure. Orotracheal intubation with mechanical ventilation was performed. A refractory shock against the measures adopted ensued, which ended up in cardio respiratory arrest and death. A post mortem hepatic biopsy was performed, producing an anatomopathological compatibility with chronic hepatitis.

\section{Discussion}

The case reported was initially seen as a compressive TM by subdural hematoma. Nevertheless, the evolution throughout the patient's stay in hospital revealed a cervical neurological decay. A new MRI was requested to assess the presence of a new hematoma in the region, which explained such evolutionary status. However, the MRI and the clinical and laboratorial evaluations suggested other likely diagnostic etiologies for myelitis as no compressive or ischemic lesion in the vertebral column was observed. A diffuse, demyelinating change in the medulla was more conspicuously observed, which was lately corroborated by the findings of the spinal fluid puncture (pleocytosis, and high levels of basic myelin protein).

TM is an acute or sub acute inflammatory condition, manifested as the implication of spinal cord functions and resulting in motor and sensory loss below the point affected in the vertebral column. As a rule, the disease is clinically observed as bilateral weakening of lower limbs, and sometimes upper limbs, accompanied by urinary and intestinal dysfunctions, if no neurological disease or compression of the spinal cord are present [10]. TM etiology is diverse, including infectious and rheumatologic processes, deficiency of vitamin B12, and multiple sclerosis [6,11].

As regards the infectious causes, several pathogens are implicated in the progression of TM, such as infection by the syphilis spirochete, by HIV, HTLV-1 and 2, cytomegalovirus, other viruses of the herpes family, and in the presence of toxoplasmosis. Yet, these diseases were ruled out from the present case, as serologies for these pathogens were all negative (Table 2). Schistosomosis is an important cause behind myelitis in zones where the parasite reaches endemic proportions, and though Brazil is considered as having this epidemiologic status, the southern region of the country falls out of these criteria, with low endemics figures. The few cases reported in the state of Rio Grande do Sul were diagnosed in the region of the municipality of Esteio [12], a place to which the patient was not exposed. Mycoplasma pneumoniae is a rare causal agent of TM, which manifests as a respiratory disease prior to the neurological status by roughly 10 days [13], and was not present in the present case study. Other possible infections were discarded, including the less common causes of MT, as influenza, measles, varicella, Lyme disease, German measles, and mumps. The clinical evaluation did not make these diseases relevant to this discussion [14]. 
Table 1. Laboratory exams results on admission.

\begin{tabular}{lll}
\hline Exams & Admission & In 4 weeks \\
Urea & $273.1 \mathrm{mg} / \mathrm{dL}$ & 24.9 \\
Creatinine & $9 \mathrm{mg} / \mathrm{dL}$ & 0.5 \\
AST & $156 \mathrm{UI} / \mathrm{l}$ & 160 \\
AST & $61 \mathrm{UI} / \mathrm{l}$ & 86 \\
Total bilirubins & $0.54 \mathrm{mg} / \mathrm{dL}$ & 0.71 \\
Amylase & 50 & - \\
TP & $35 \%$ & $48 \%$ \\
TTP & $32 \mathrm{~s}$ & $108 \mathrm{~s}$ \\
INR & 1.8 & \\
Albumin & 3.2 & 0.60 \\
C-reactive protein & & 8.7 \\
Hemoglobin & $13.1 \mathrm{mg} / \mathrm{dL}$ & 9800 \\
Leukogram & 44900 & - \\
Metamyelocytes & $1 \%$ & - \\
Band forms & $22 \%$ & 73.8 \\
Segmented eosinophils/neutrophils & $63 \%$ & 15.5 \\
Lymphocytes & 5.5 & 9.3 \\
Monocytes & $8 \%$ & 1.0 \\
Eosinophils & $0 \%$ & 172 \\
Platelets & $328.000 / \mathrm{mm}^{3}$ & \\
\hline
\end{tabular}

Table 2. Immunological, microbiological, cytological and biochemical analyses of the cerebrospinal fluid after a sub occipital puncture.

\begin{tabular}{lcc}
\hline Exams & Result & Reference values \\
\hline Anti-HIV 1 and 2 & Not Reagent & \\
HbsAg & NR & \\
Anti-HCV serum & Reagent & Not Reagent \\
VDRL & Reagent & \\
Cytomegalovirus IgG & Not Reagent & \\
Cytomegalovirus IgM & Not Reagent & \\
Toxoplasmosis IgG & Not Reagent & \\
Toxoplasmosis IgM & Reagent & \\
Herpes 1 and 2 IgG & Not Reagent & \\
Herpes 1 and 2 IgM & Not Reagent & \\
HTLV 1 and 2 & Not Reagent & $10-40$ \\
FAN & Not Reagent & $0.27-4.2$ \\
pANCA & Not Reagent & \\
cANCA & $>1000$ & \\
Vitamin B12 & 121.9 & \\
C3 & 19.5 & \\
C4 & 1.57 & $<6$ \\
TSH & 17.6 & \\
Protein in spinal fluid & Reagent & \\
Anti HCV in spinal fluid & 173.8 & \\
Glucose in spinal fluid & 18.6 & \\
Cellularity in spinal fluid & 3.5 & \\
DHL in spinal fluid & 15.5 & \\
Adenosine Deamynase in spinal fluid & $90 \%$ neutron) & \\
Basic myelin protein & & \\
\hline & &
\end{tabular}


Figure 1. MRI of the encephalon does not reveal the presence of lesions.
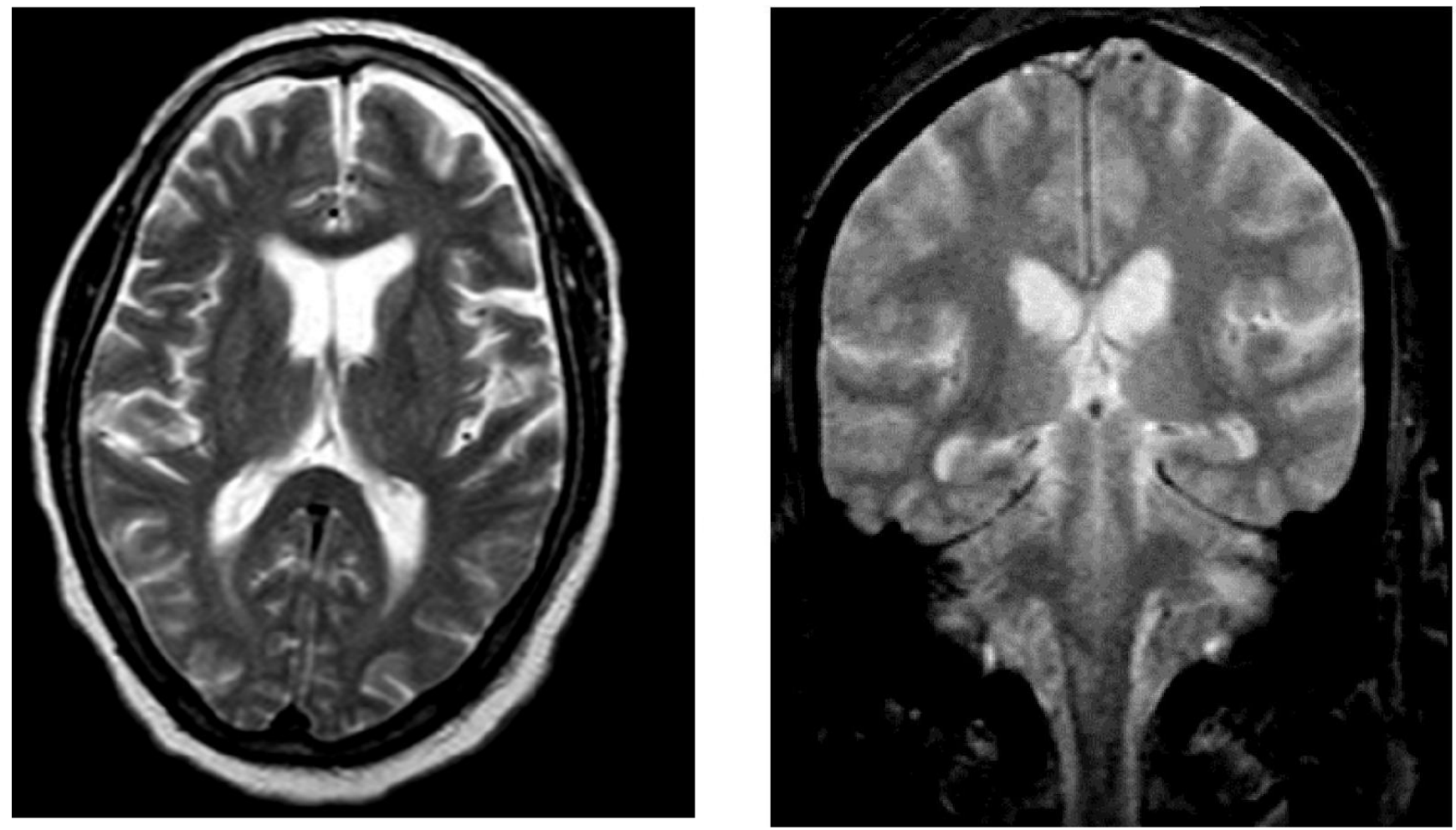

Figure 2. MRI of cervico-dorsal vertebral column, sagittal plane with intramedullary hyposignal in T1, extensive hypersignal in T2 in the medulla, starting at the bulbomedullar transition and stretching to the visible sections of the dorsal medulla, without significantly increasing medullar thickness.
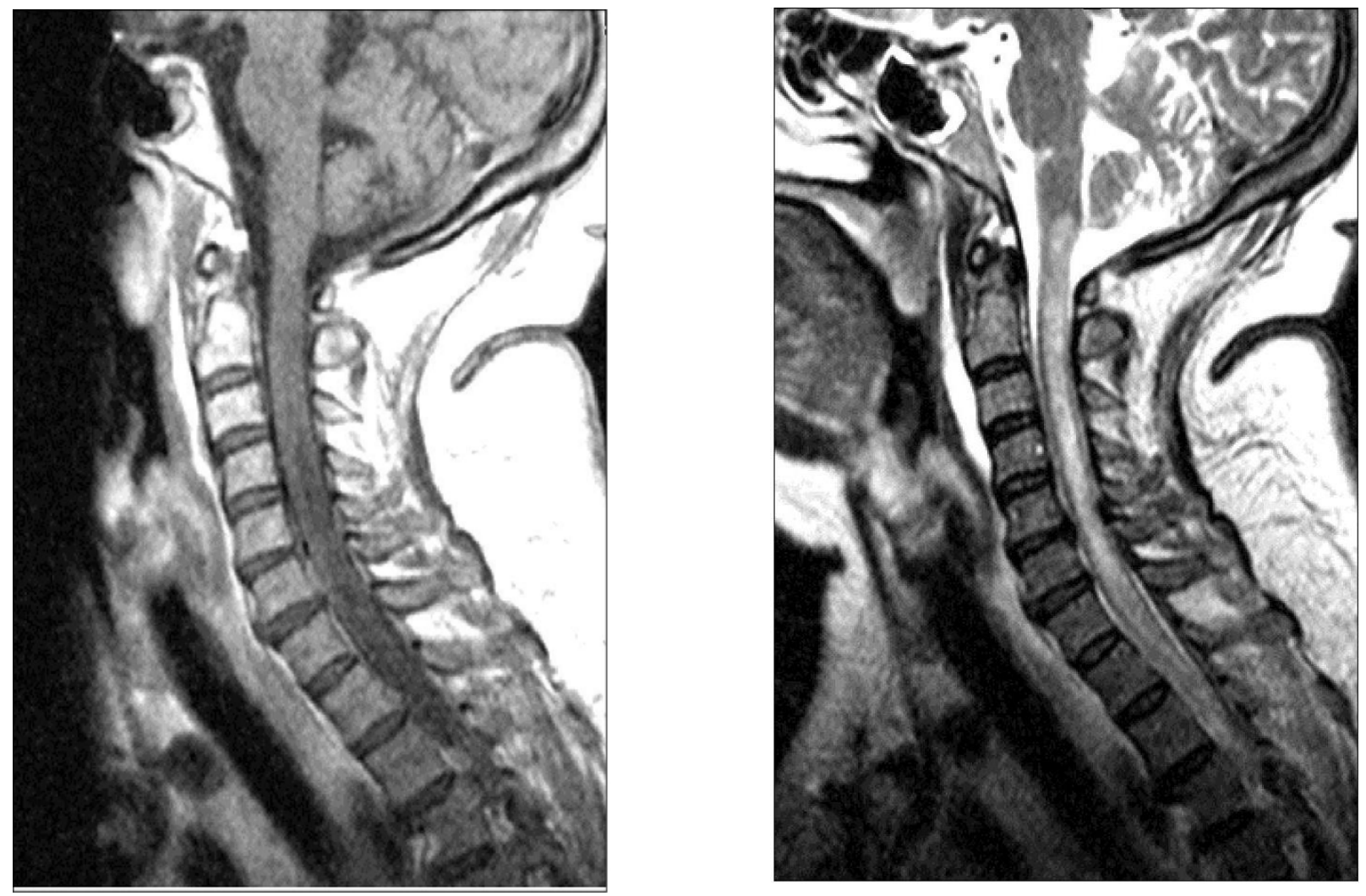
The rheumatologic etiologies involved in the progression of TM are systemic lupus erythematosus, antiphospholipid syndrome, Sjögren's syndrome, Behcet syndrome [11], and sarcoidosis. These diseases were ruled out from this case report, as the patient did not present symptoms or laboratory findings to meet the diagnostic criteria for these diseases. Essential mixed cryoglobulinaemia, which relates to HCV infection and may even be present in $44 \%$ to $54.3 \%$ of patients with chronic hepatic disease caused by the virus $\mathrm{C}[15,16]$, even in asymptomatic patients, was not tested in the present case study, and thus ruled out. This decision was taken based on the fact that the patient did not present other changes, such as purpura, kidney parenchyma disease, and low serum complement level. Cryoglobulinaemia has been traditionally linked to syndromes such as peripheral neuropathy, multiple mononeuritis and polyradiculoneuropathy, and does not establish the causal relationship between TM and this vasculitis.

Multiple sclerosis is a heterogeneous disease, whose manifestations may be acute bouts, chronic progressive, or remittent and recurrent, as typically observed. Even in the isolated syndromic manifestations, as TM and optic mononeuritis, it is estimated that between $50 \%$ and $80 \%$ of these episodes may show altered cranium MRI findings, with a negative predictive value of $86 \%$, which rules out multiple sclerosis from this diagnostic picture [17].

The relationship between myelitis and HCV, with currently limited but increasing evidences, is being more consistently proved. The relationship was first described by ZandmanGoddard in 2003, in a case report of a 34-year-old male patient with chronic HCV infection. The patient developed TM with paraplegia, and a hyper signal at the spinal cord in D4-5 visualized by MRI. Grewal et al. described a case of recurrent myelitis in a 46-year-old male patient with chronic HCV infection, in a case report in which a medulla biopsy revealed acute demyelination with no evidence of vasculitis. Anti-HCV antibodies were present in the spinal fluid, though the PCRRNA investigation was not detected in neither patient, which points to an immune mediated pathogenesis.

The present case study describes the detection of anti$\mathrm{HCV}$ in the spinal fluid using the ELISA protocol, which lends cogency to the hypothesis of HCV-mediated myelitis, as seen in the case studies exemplified above. It was not possible to detect PCR-RNA in the spinal fluid, as we did not have the means to an appropriate technique for its detection, though in no case described above HCV was detected by PCR-DNA. The absence of HCV in the spinal fluid does not rule out the virus involvement as etiologic agent, but such absence makes the immune-mediated lesion hypothesis more consistent, and not caused by the direct action of the virus.

Recently, Aktipi et al. described a series of seven patients with chronic HCV infection, all presenting recurrent TM and drawn from a cohort study with 59 patients with TM not caused by multiple sclerosis, and for whom MRI ruled out compressive, vascular and actinic myelopathy. In that cohort study, 21 (36\%) patients presented at least one recurrence of the disease, and were classified as having recurring TM. The seven HCV-positive patients represented $12 \%$ of the myelitis cases as a whole, and $33 \%$ of the recurrent cases. Among these, in the time the first episode occurred, only one patient had been identified previously as positive for $\mathrm{HCV}$, and only one presented changes in the hepatic inflammatory findings [18].

$\mathrm{HCV}$ is responsible for a large spectrum of autoimmune phenomena, possibly as a result of the chronic viral stimulus, which is believed to be able to determine a direct response against blood vessels and/or myelin, thus leading to TM. The presence of anti-HCV antibodies in the spinal fluid corroborates this pathogen as well as a likely lesion directly related to the virus $[9,18]$. At the moment, the evidences of implications in the spinal cord that manifest as TM are being associated to chronic HCV infection, especially in the recurrent forms of the disease, ruling out other likely etiologies.

Thus, the present case report suggests the inclusion of $\mathrm{HCV}$ investigation in the initial diagnosis of TM. It is important to recognize and identify the likely neurological changes involving the spinal cord due to the presence of the pathogen. This approach makes possible to perform an early diagnosis, as in the cases described a positive response has been observed with the immunosuppressant treatment with corticoids, though the data are still scarce to define the treatment management and prognosis.

\section{Acknowledgements}

Dr. Renato Fagundes and Dr Alexandre Vargas Schwarzbold, we especially thank for the given support and the repeated reviews and opinions, who when requested always demonstrated availability.

\section{References}

1. World Health Organization, 2002, Hepatitis C. Available on < http://www.who.int/csr/disease/hepatitis/whocdscsrlyo2003/en/> Had Access day: 24 june 2007.

2. Bichuetti D.B., Oliveira A.S.B. Manifestações Neurológicas nas Hepatites Virais. Ver Neurociências 2005;13(3):133-7.

3. Tembl J.I., Ferrer J.M., Sevilla M.T., et al. Neurologic complications associated with hepatitis C virus infection. Neurology 1999;53(4):861-4.

4. Bolay H., Söylemezoglu F., Nurlu G., et al. PCR detected hepatitis virus genome in brain of a case with progressive encephalomyelitis with rigity. Clin Neurol Neurosurg 1996;98(4):305-8.

5. Morgello S. The Nervous Systen and Hepatitis C; Semin Liver Dis. 2005;25(1):118-21.

6. Transverse Myelitis Consortium Working Group. Proposed diagnostic criteria and nosology of acute transverse Myelitis. Neurology 2002;54:499-505.

7. Brito J.C.F., Nobrega P.V. Mielopatias considerações clínicas e aspectos etiológicos; Arq Neuropsiquiatr 2003;61(3-B):816-21.

8. Zandman-Goddard G., Levy Y., Weiss P., et al. Transverse myelitis associated with chronic hepatitis C. Clin Exp Rheumatol 2003;21(1):111-3.

9. Grewal A.K., Lopes M.B., Berg C.L., et al. Recurrent demyelinating myelitis associated with hepatitis C viral infection.J Neurol Sci 2004;224(1-2):101-6.

10. Scotti G., Gerevini S. Diagnosis and differential diagnosis of acute transverse myelopathy. The role of neuroradiological investigations and review of the literature; Neurol Sci 2001;22:S69-S73. 
11. Theodoridou A., Settas L. Demyelination in rheumatic diseases. J Neurol Neurosurg Psychiatry 2006;77(3):290-5.

12. Caldeira K.V., Rangel S., Costa I.A. Controle da Esquistossomose em esteio e Municípios limítrofes, ano 2004; Boletim. Epidemiológico v. 7, n. 2, junho, 2005; Secretária de Saúde do RS-Brasil.

13. Tsiodras S., Kelesidis T., Kelesidis I., et al. Mycoplasma pneumoniae-associated myelitis: a comprehensive review. Eur J Neurol 2006;13(2):112-24.

14. Hauser S.L., Ropper A.H. Doenças da medulla espinhal. In: Kasper D.L., Braunwald E., Fauci A.S., et al. [eds]. Harrison Medicina Interna, Decima sexta edição Trad. Rio de Janeiro: Editora MacGrawHill Interamericana do Brasil, 2006 Volume 2:2558-67.
15. Lunel F., Musset L., Cacoub P., et al. Cryoglobulinemia in chronic liver diseases: role of hepatitis $\mathrm{C}$ virus and liver damage. Gastroenterology 1994;106(5):1291-300.

16. Kayali Z., Buckwold V.E., Zimmerman B., Schmidt W.N. Hepatitis C, cryoglobulinemia, and cirrhosis: a metaanalysis. Hepatology 2002;36(4 Pt 1):978-85.

17. Arnold D.L., Matthews P.M. MRI in the diagnosis and management of multiple sclerosis. Neurology 2002;58(8 Suppl 4):S23-31.

18. Aktipi K.M., Ravaglia S., Ceroni M., et al. Severe recurrent myelitis in patients with hepatitis C virus infection; Neurology 2007;68(6):468-9. 